Systematic Review

\title{
Comparative Effectiveness Review of Cooled Versus Pulsed Radiofrequency Ablation for the Treatment of Knee Osteoarthritis: A Systematic Review
}

Anita Gupta, DO, PharmD', Daniel P. Huettner, BS ${ }^{1,2}$, and Matthew Dukewich, PharmD ${ }^{2}$

From: ${ }^{1}$ Department of Anesthesiology and Perioperative Medicine, Drexel University College of Medicine, Philadelphia,

PA; ${ }^{2}$ Drexel University

College of Medicine, Philadelphia, PA

Address Correspondence: Anita Gupta, DO, PharmD 245 North 15th St., New College Building, MS 310

Philadelphia, PA 19102 Email: anita.gupta@drexelmed.

edu

Disclaimer: There was no external funding in the preparation of this manuscript.

Conflict of interest: Each author certifies that he or she, or a member of his or her immediate family, has no commercial association

(i.e., consultancies, stock ownership, equity interest, patent/licensing arrangements, etc.) that

might pose a conflict of interest in connection with the submitted manuscript.

Manuscript received: o8-12-2016

Revised manuscript received: 10-06-2016 Accepted for publication: 10-26-2016

Free full manuscript: www.painphysicianjournal.
Background: Patients suffering from osteoarthritis of the knee and patients post total knee arthroplasty often develop refractory, disabling chronic knee pain. Radiofrequency ablation, including conventional, pulsed, and cooled, has recently become more accepted as an interventional technique to manage chronic knee pain in patients who have failed conservative treatment or who are not suitable candidates for surgical treatment.

Objective: This systematic review aimed to analyze published studies on radiofrequency ablation to provide an overview of the current knowledge regarding variations in procedures, nerve targets, adverse events, and temporal extent of clinical benefit.

Study Design: A systematic review of published studies investigating conventional, pulsed, or cooled radiofrequency ablation in the setting of chronic knee pain.

Methods: Medline, Google Scholar, and the Cochrane Central Register of Controlled Trials (CENTRAL) databases were reviewed for studies on radiofrequency ablation for patients with chronic knee pain through July 29, 2016. From the studies, the procedural details, outcomes after treatment, follow-up points, and complications were compiled and analyzed in this literature review. Included studies were analyzed for clinical relevance and strength of evidence was graded using either the NHLBI Quality assessment of controlled intervention studies or the NHLBI quality assessment for before-after (pre-post) studies with no control group.

Results: Seventeen total publications were identified in the search, including articles investigating conventional, pulsed, or cooled radiofrequency ablation. These studies primarily targeted either the genicular nerves or used an intraarticular approach. Of the studies, 5 were small-sized randomized controlled trials, although one involved diathermy radiofrequency ablation. There were 8 retrospective or prospective case series and 4 case reports. Utilizing the strength of evidence grading, there is a low level of certainty to suggest a superior benefit between targeting the genicular nerve, an intraarticular approach, or targeting the larger nerves such as femoral and tibial nerves. Utilizing the strength of evidence grading, there is a low level of certainty in supporting the superiority of any specific RFA procedure modality. The majority of the studies report positive patient outcomes, but the inconsistent procedural methodology, inconsistent patient assessment measures, and small study sizes limit the applicability of any specific study to clinical practice.

Limitations: While the wide search strategy included a variety of articles, broad conclusions and pooled data could not be obtained based on the studies analyzed.

Conclusions: Overall, the studies showed promising results for the treatment of severe chronic knee pain by radiofrequency ablation at up to one year with minimal complications. Numerous studies, however, yielded concerns about procedural protocols, study quality, and patient follow-up. Radiofrequency ablation can offer substantial clinical and functional benefit to patients with chronic knee pain due to osteoarthritis or post total knee arthroplasty.

Key words: Radiofrequency ablation, knee osteoarthritis, knee pain, genicular nerve, total knee arthroplasty (TKA), cooled radiofrequency ablation, pulsed radiofrequency ablation

Pain Physician 2017; 20:155-171 
O steoarthritis (OA) is one of the most prevalent chronic diseases in the United States with an estimated nearly 40 million Americans impacted (1). OA of the knee is a leading cause of disability in the United States, affecting nearly one in 10 older adults with an annual prevalence of radiographic evidence of knee OA of greater than $37 \%$ (2). OA of the knee is one of the top 5 leading causes of impairment among adults - an estimated $80 \%$ of patients with knee OA have some movement limitation, while $25 \%$ are unable to perform major activities of daily living (3). Clinically, patients with knee OA typically present with a chief complaint of pain, often associated with limited range of motion, stiffness, osteophytes, crepitus, and effusions. Due to the progressive degenerative nature of OA and the associated pain, patients become more physically impaired through the course of the disease. Unfortunately, because no cure has been established for OA, treatment currently focuses on improving the quality of life for patients through pain control, decreasing physical impairment, and slowing the progression of the disease (4). Treatment options for patients with OA include conservative approaches, such as weight loss, physical therapy, and pharmacological interventions, while the more invasive approaches include intraarticular injections, joint preserving surgical treatment, and total knee arthroplasty (TKA). Despite these treatment options, patients with severe OA of the knee often suffer from refractory pain and look for other modalities besides surgical interventions $(5,6)$.

Radiofrequency ablation (RFA) is a novel technique used to treat a wide variety of cancers, cardiac arrhythmias, and other numerous conditions, but it has also recently gained popularity in alleviating chronic pain in patients with musculoskeletal disorders such as OA (7). The minimally invasive conventional technique involves a high temperature probe that targets specific nerves innervating the tissue of interest. In the case of knee OA, RFA was first introduced in 2010 by Choi et al and further explored in subsequent years $(8,9)$. In addition to the conventional method, other methods of RFA for knee OA include pulsed and cooled RFA.

\section{Relevant Anatomy}

Neuronal innervation of the knee is substantially intricate. The knee is innervated by the articular branches, known as the genicular nerves, of several major nerves, including the femoral, tibial, common peroneal (fibular), saphenous, and obturator nerves $(10,11)$. More specifically, the tibial nerve provides branches that innervate the articular capsule as they follow the superior medial and superior lateral vascular supplies $(11,12)$. In the same way, the common peroneal nerve provides 2 articular branches that innervate the inferolateral articular capsule, while another common peroneal branch innervates the anterolateral as it follows the interior lateral genicular vasculature $(11,12)$. A study of 8 cadaver knee dissections showed similar findings, the superolateral branch from the vastus lateralis, superomedial branch from the vastus medialis, middle branch from the vastus intermedius, inferolateral (recurrent) branch from the common peroneal nerve, inferomedial branch from the saphenous nerve, and a lateral articular nerve branch from the common peroneal nerve (13). Thus, RFA requires identification of anatomical landmarks around the knee to locate the various genicular nerve branches that innervate the joint. As a result, the superomedial, superolateral, and inferomedial genicular nerve branches are commonly targeted by their proximal relation to bony landmarks $(8,12)$.

\section{Methods}

The current literature review was conducted through a PubMed search up to July 29, 2016, using the following terms: "radiofrequency," "osteoarthritis," "knee," and "pain." The year of the study, study design, size, mean age of patients, RFA methodology, nerve target, follow-up time, outcome scoring method, study results, and adverse events were reported. A Medline search using "radiofrequency," "knee," and "pain" returned 23 results. A Medline search of "radiofrequency," "osteoarthritis," and "knee" returned 34 results. A Cochrane Central Register of Controlled Trials (CENTRAL) search of "radiofrequency" and "knee" returned 26 trials, including 4 relevant trials and 2 new relevant ones, but no peer-reviewed publications were found for these 2 new trials.

The references of every publication were reviewed in order to identify any additional articles that were not found in the original search. With the Medline and CENTRAL searches and review of references, a total of 17 studies were considered for this review. The degree of evidence regarding the efficacy of radiofrequency ablation in the literature was not substantially high - only 5 small-sized randomized controlled trials were identified with size ranging from 17 to 54 patients $(6,8,14$ 16). The remaining studies were retrospective (17-20) and prospective (21-24) observational case series and case reports $(12,25-27)$. Studies were included in this re- 
view if they met the following inclusion criteria: studies should define criteria for qualifying for RFA treatment procedures such that analogous patients can be identified in other practice environments; specific intervention should be described in enough detail to replicate in relevant patients or to allow comparison to similarly described interventions, including nerve target, imaging modality used, and RFA strategy; if a comparator treatment is utilized in the study, it should be described in enough detail as to be replicated in a relevant population and it should be a clinically acceptable choice for the level of pain targeted by the intervention; study should include relevant outcomes, including duration of follow-up, pain scores before and after intervention, quality of life measures, and any adverse events reported. The complete list of studies analyzed for this review is outlined in Table 1 . Included studies were analyzed first for clinical relevance, with the grading scale shown in Table 2 and the results shown in Table 3. Individual studies were then graded for strength. Controlled intervention studies were graded according to criteria in Table 4, while case and case series studies were graded according to criteria in Table 5. Both sets of criteria were based on methodology developed by the National Heart, Lung, and Blood Institute (NHLBI). Results of this grading process is reported in Table 6 and Table 7. The overall strength of evidence for the RFA procedures analyzed is reported according to the United States Preventive Services Task Force levels of certainty regarding net benefit (Table 8).

\section{Conservative Treatment Modalities}

The primary objective in clinically managing patients with OA of the knee involves controlling pain and swelling, improving quality of life, improving physical capacity, and preventing progression of the arthritis. Initially, patients should be treated with more conservative approaches, such as physical therapy, orthoses, exercise programs, or weight loss, before pharmacological intervention is initiated. Obesity has a strong correlation to the development of $\mathrm{OA}$ and weight loss programs have significant impacts on the development and progression of OA (28-30). One study found that the probability of developing OA of the knee was decreased by $50 \%$, in a linear fashion, with a 10 pound weight loss over 10 years (30). Diet and exercise regimens are even more beneficial; several trials demonstrated that the combination of diet and exercise improved quality of life, knee pain, and mobility among patients with knee OA $(31,32)$. Weight loss goals, however, may take a sig- nificant amount of time to achieve, and patients with severe pain require approaches that provide immediate relief in order to improve their quality of life.

Physical therapy, which aims to strengthen supporting muscle groups and improve flexibility, is a mainstay therapy for OA, as it has demonstrated significant symptomatic relief for patients and improved clinical outcomes. Patients report pain relief, decreased stiffness, and improved mobility through physical therapy (33-36). Furthermore, physical therapy may delay the need for surgical intervention - one study found a $15 \%$ decrease in one year rates of TKA for patients with OA who participated in physical therapy (37). Studies support an integrated exercise program approach as part of the multimodal treatment for knee OA $(38,39)$. Tai chi, which is a traditional Chinese technique combining awareness exercises, deep breathing exercises, movement, and meditation, is another therapeutic option that can be designed specifically for OA patients to provide symptomatic relief (40). Patients with moderate to severe OA of the knee, however, may experience minimal benefit from physical therapy and exercise programs compared to patients with mild OA, as shown in a study of patients undergoing short-term, high-intensity exercise programs (41). For patients with severe OA, aquatic exercise can provide benefit as the water's buoyancy decreases joint compression and permits exercises that would otherwise be limited $(42,43)$. Unsurprisingly, another concern for the efficacy of physical therapy and exercise programs is patient compliance; consequently, supervised settings increase the likelihood of patient benefit (44). Nevertheless, exercise and physical activity has clearly been shown to be beneficial to the patient and reduces allcause mortality, justifying its implementation in every patient's treatment plan if possible (45).

Patients who have not responded appropriately from nonpharmacological treatment are candidates for medical management of their knee OA. Medications used in the pharmacological approach primarily include analgesics, nonsteroidal anti-inflammatory drugs (NSAIDS), and intraarticular glucocorticoids. Initial drug treatment with acetaminophen is recommended as the first-line approach, followed by NSAIDS (46). One study, however, found no role for paracetamol (acetaminophen) in the treatment of OA (47). Non-selective NSAIDS (e.g., ibuprofen, diclofenac, or naproxen) and COX-2 specific NSAIDS (celecoxib) have demonstrated efficacy through numerous studies in their treatment of knee OA (47-49). NSAIDS should be used with cau- 
Pain Physician: March/April 2017: 20:155-171

\begin{tabular}{|c|c|c|c|c|c|c|c|c|c|c|}
\hline 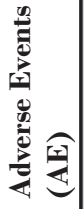 & $\frac{\ll}{z}$ & 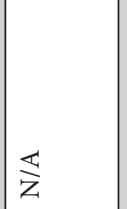 & 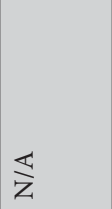 & 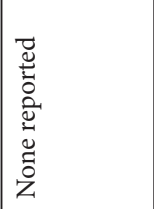 & $\overleftrightarrow{\nwarrow}$ & 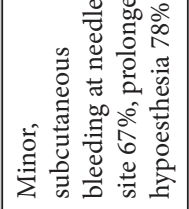 & 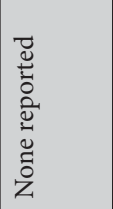 & 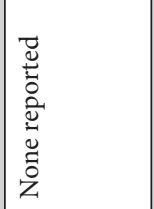 & 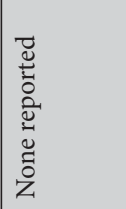 & $\overleftrightarrow{\zeta}$ \\
\hline 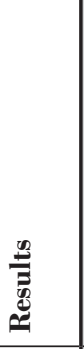 & 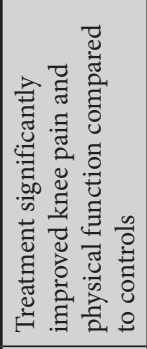 & 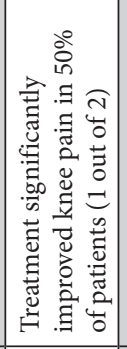 & 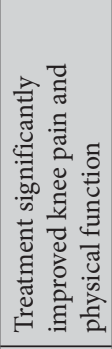 & 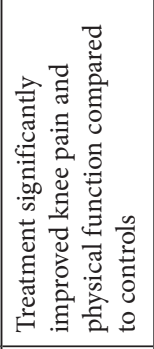 & 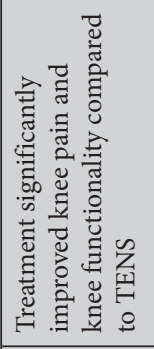 & 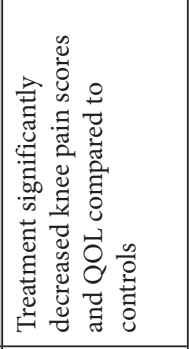 & 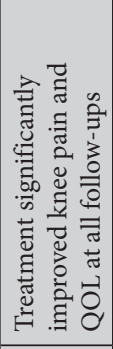 & 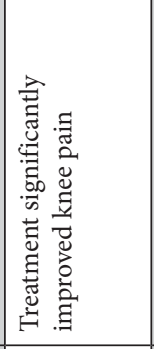 & 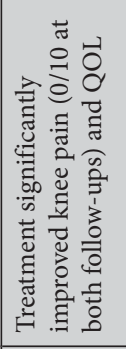 & 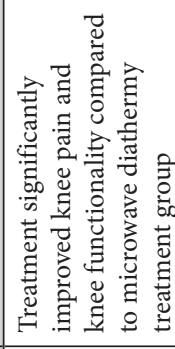 \\
\hline 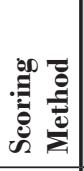 & 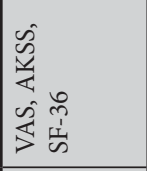 & $\sum_{i}^{\infty}$ & $\begin{array}{l}0 \\
0 \\
0 \\
0 \\
0 \\
5 \\
1 \\
5\end{array}$ & 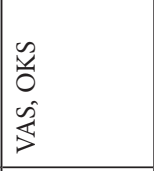 & 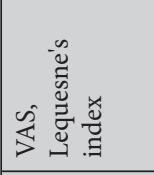 & 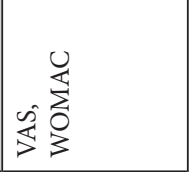 & 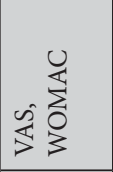 & $\begin{array}{l}0 \\
0 \\
0 \\
0 \\
5\end{array}$ & 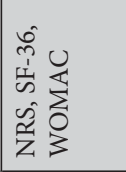 & 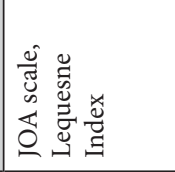 \\
\hline 言 & ‡ & $\begin{array}{l}\stackrel{8}{\Xi} \\
\stackrel{1}{I} \\
1 \\
= \\
=\end{array}$ & $\stackrel{\Xi}{m}$ & 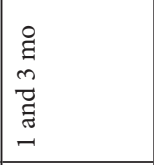 & 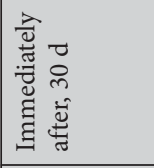 & $\begin{array}{l}0 \\
\vdots \\
0 \\
\tilde{m} \\
-1\end{array}$ & $\begin{array}{l}\approx \\
0 \\
\hat{m} \\
-=\end{array}$ & $\begin{array}{l}0 \\
\vdots \\
a \\
6 \\
\hat{m} \\
-1\end{array}$ & $\begin{array}{l}0 \\
\vdots \\
0 \\
0 \\
0 \\
3 \\
0 \\
0\end{array}$ & $\begin{array}{l}\frac{w}{3} \\
m \\
-\overrightarrow{0} \\
\text { a } \\
-1\end{array}$ \\
\hline 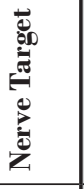 & $\overleftrightarrow{\Sigma}$ & 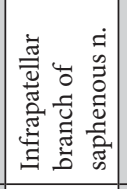 & 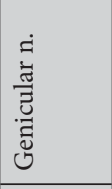 & 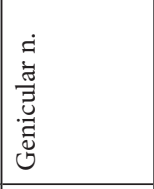 & $\overleftrightarrow{\breve{z}}$ & 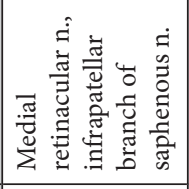 & 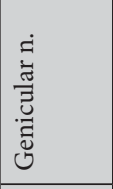 & 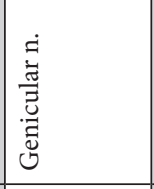 & 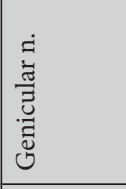 & $\mathbb{z}$ \\
\hline 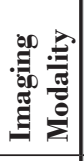 & ‡ั & 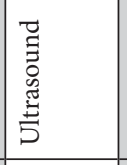 & 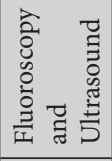 & 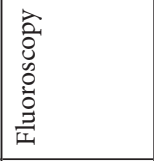 & हั & ह̆ & 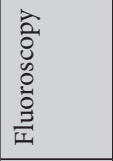 & 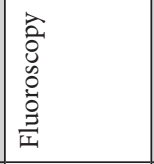 & 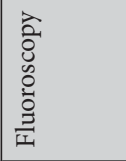 & 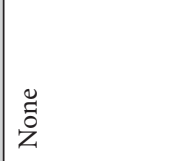 \\
\hline 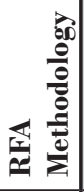 & 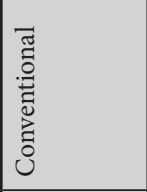 & 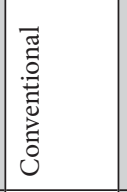 & 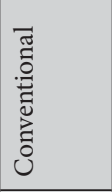 & 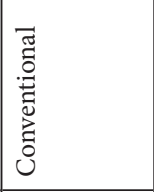 & 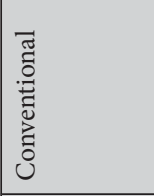 & 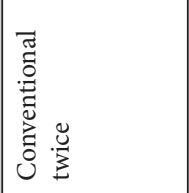 & 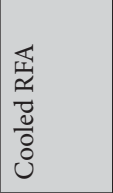 & 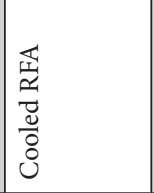 & 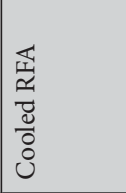 & 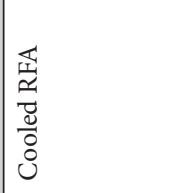 \\
\hline 䨌 & 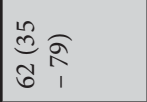 & $\begin{array}{ll}\vec{m} & \widehat{\delta} \\
\sigma & 0\end{array}$ & $\stackrel{\infty}{+}$ & 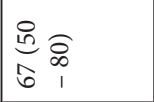 & 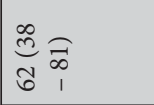 & 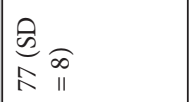 & 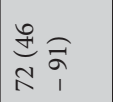 & $\approx$ & $\vec{\infty}$ & 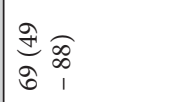 \\
\hline 竞 & in & 4 & - & $\stackrel{\infty}{m}$ & 아 & $\stackrel{m}{m}$ & $a$ & - & - & $=$ \\
\hline 总 & 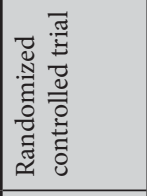 & 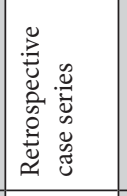 & 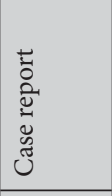 & 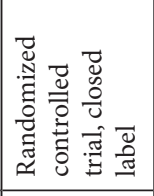 & 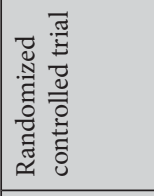 & 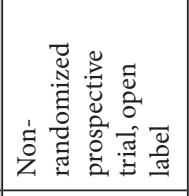 & 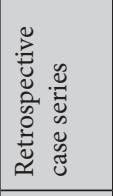 & 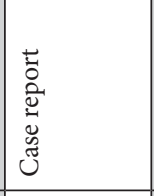 & 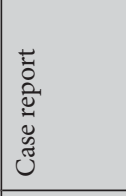 & 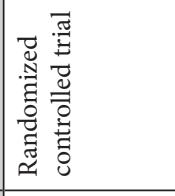 \\
\hline 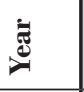 & 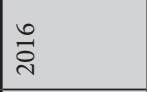 & $\stackrel{n}{\stackrel{n}{n}}$ & 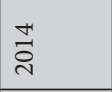 & $\overrightarrow{\tilde{\sim}}$ & 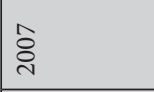 & $\overrightarrow{\tilde{\sim}}$ & $\stackrel{n}{\stackrel{2}{2}}$ & 苜 & $\stackrel{0}{\stackrel{\sim}{\pi}}$ & 茓 \\
\hline 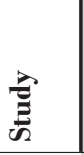 & 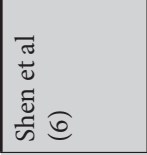 & 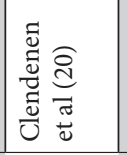 & 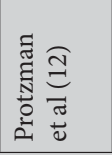 & 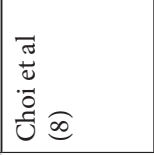 & 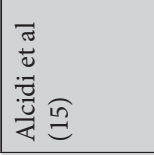 & 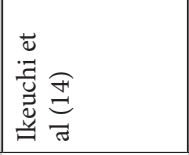 & 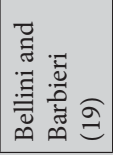 & 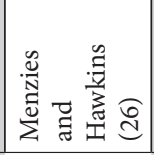 & 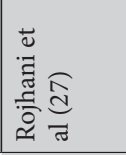 & 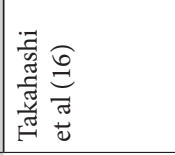 \\
\hline
\end{tabular}


Effectiveness Review of Cooled Versus Pulsed Radiofrequency Ablation

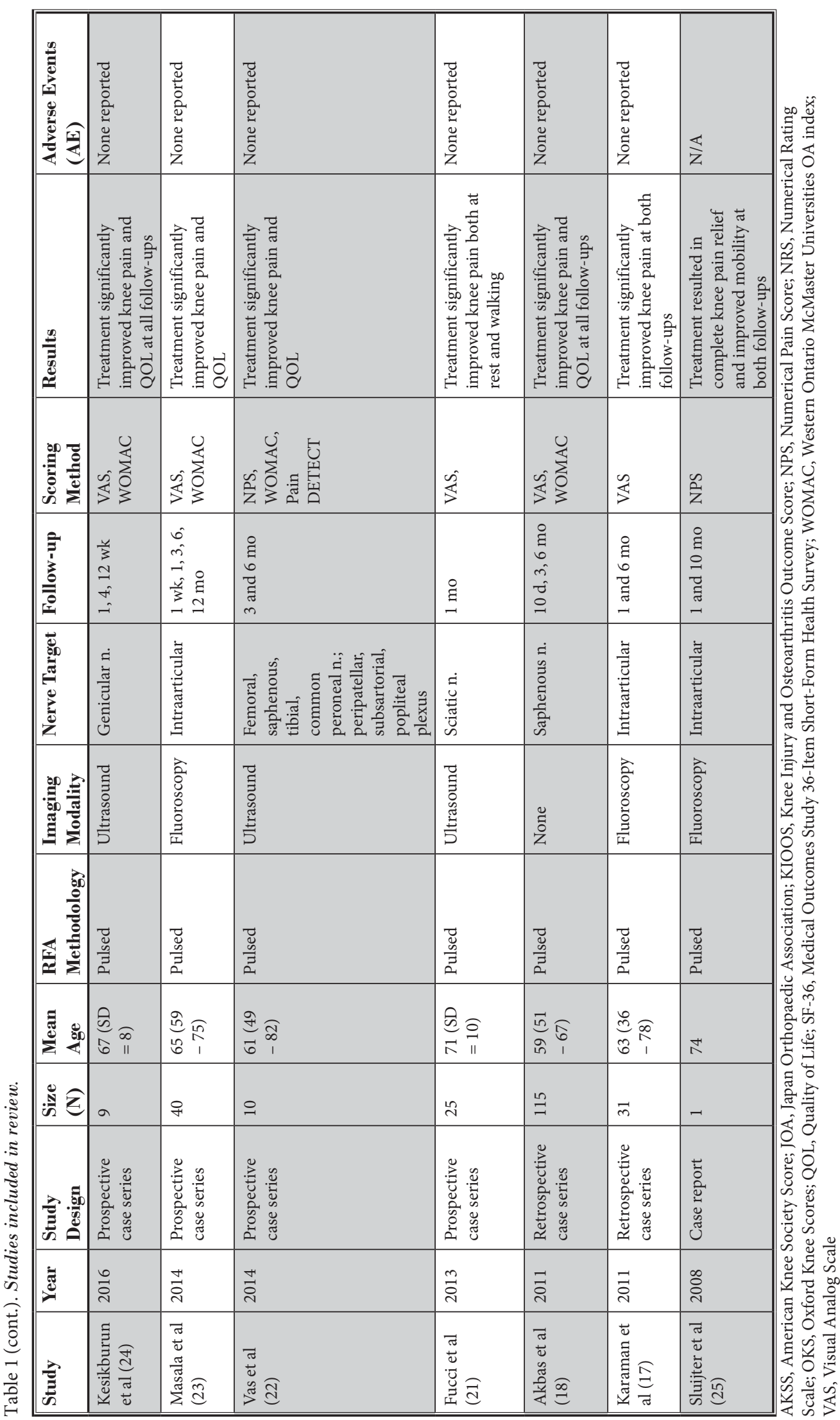


Table 2. Clinical relevance scale (adapted from 86 and 87 ).

\begin{tabular}{|l|l||}
\hline & $\begin{array}{l}\text { Yes (+)/No (-)/ } \\
\text { Uncertain (U) }\end{array}$ \\
\hline A.) Are the patients described in detail to allow comparison to patients seen in similar interventional pain practices? & \\
\hline B.) Are the interventions and treatment settings described with enough clarity to replicate in a similar practice? & \\
\hline C.) Were clinically relevant outcomes measured and reported? & \\
\hline D.) Is the size of the effect clinically important? & \\
\hline E.) Do the benefits of treatment outweigh potential and reported harm? & \\
\hline
\end{tabular}

Table 3. Clinical relevance grading of included studies.

\begin{tabular}{|c|c|c|c|c|c|c|}
\hline Manuscript & A) Patient & B) Interventions & C) Outcomes & D) Effect size & E) Benefit vs harm & Grade \\
\hline Shen et al, 2016 (6) & + & + & + & + & $\mathrm{U}$ & $4 / 5$ \\
\hline Clendenen et al, 2015 (20) & + & + & + & + & $\mathrm{U}$ & $4 / 5$ \\
\hline Protzman et al, 2014 (12) & + & + & + & - & + & $3 / 5$ \\
\hline Choi et al, 2011 (8) & + & + & + & + & + & $5 / 5$ \\
\hline Alcidi et al, 2007 (15) & - & + & + & + & $\mathrm{U}$ & $3 / 5$ \\
\hline Ikeuchi et al, 2011 (14) & + & + & + & + & + & $5 / 5$ \\
\hline Bellini and Barbieri, 2015 (19) & + & + & + & + & + & $5 / 5$ \\
\hline Menzies and Hawkins, 2015 (26) & + & + & + & + & + & $5 / 5$ \\
\hline Rojhani et al, 2016 (27) & + & + & + & + & + & $5 / 5$ \\
\hline Takahashi et al, 2016 (16) & + & + & + & + & $\mathrm{U}$ & $4 / 5$ \\
\hline Kesikburun et al, 2016 (24) & + & + & + & + & + & $5 / 5$ \\
\hline Masala et al, 2014 (23) & + & + & + & + & + & $5 / 5$ \\
\hline Vas et al, 2014 (22) & + & + & + & + & + & $5 / 5$ \\
\hline Fucci et al, 2013 (21) & + & + & + & + & + & $5 / 5$ \\
\hline Akbas et al, 2011(18) & + & + & + & + & + & $5 / 5$ \\
\hline Karaman et al, 2011 (17) & + & + & + & + & + & $5 / 5$ \\
\hline Sluijter et al, 2008 (25) & + & + & - & $\mathrm{U}$ & $\mathrm{U}$ & $2 / 5$ \\
\hline
\end{tabular}

Yes (+) / No (-) / Uncertain (U)

Table 4. NHLBI quality assessment of controlled intervention studies (88).

\begin{tabular}{|c|c|c|c|}
\hline Criteria & Yes & No & $\begin{array}{l}\text { Other (CD, } \\
\text { NR, NA)* }\end{array}$ \\
\hline 1. Was the study described as randomized, a randomized trial, a randomized clinical trial, or an RCT? & & & \\
\hline 2. Was the method of randomization adequate (i.e., use of randomly generated assignment)? & & & \\
\hline 3. Was the treatment allocation concealed (so that assignments could not be predicted)? & & & \\
\hline 4. Were study participants and providers blinded to treatment group assignment? & & & \\
\hline 5. Were the people assessing the outcomes blinded to the participants' group assignments? & & & \\
\hline $\begin{array}{l}\text { 6. Were the groups similar at baseline on important characteristics that could affect outcomes (e.g., } \\
\text { demographics, risk factors, co-morbid conditions)? }\end{array}$ & & & \\
\hline 7. Was the overall drop-out rate from the study at endpoint $20 \%$ or lower of the number allocated to treatment? & & & \\
\hline 8. Was the differential drop-out rate (between treatment groups) at endpoint 15 percentage points or lower? & & & \\
\hline 9. Was there high adherence to the intervention protocols for each treatment group? & & & \\
\hline 10. Were other interventions avoided or similar in the groups (e.g., similar background treatments)? & & & \\
\hline 11. Were outcomes assessed using valid and reliable measures, implemented consistently across all study participants? & & & \\
\hline $\begin{array}{l}\text { 12. Did the authors report that the sample size was sufficiently large to be able to detect a difference in the main } \\
\text { outcome between groups with at least } 80 \% \text { power? }\end{array}$ & & & \\
\hline 13. Were outcomes reported or subgroups analyzed prespecified (i.e., identified before analyses were conducted)? & & & \\
\hline $\begin{array}{l}\text { 14. Were all randomized participants analyzed in the group to which they were originally assigned, i.e., did they } \\
\text { use an intention-to-treat analysis? }\end{array}$ & & & \\
\hline
\end{tabular}


Table 5. NHLBI quality assessment for before-after (pre-post) studies with no control group (89).

\begin{tabular}{|c|c|c|c|}
\hline Criteria & Yes & No & $\begin{array}{c}\text { Other } \\
(\mathrm{CD}, \mathrm{NR}, \mathrm{NA}) *\end{array}$ \\
\hline \multicolumn{4}{|l|}{ 1. Was the study question or objective clearly stated? } \\
\hline \multicolumn{4}{|l|}{ 2. Were eligibility/selection criteria for the study population prespecified and clearly described? } \\
\hline \multicolumn{4}{|l|}{$\begin{array}{l}\text { 3. Were the participants in the study representative of those who would be eligible for the test/service/ } \\
\text { intervention in the general or clinical population of interest? }\end{array}$} \\
\hline \multicolumn{4}{|l|}{ 4. Were all eligible participants that met the prespecified entry criteria enrolled? } \\
\hline \multicolumn{4}{|l|}{ 5. Was the sample size sufficiently large to provide confidence in the findings? } \\
\hline \multicolumn{4}{|l|}{ 6. Was the test/service/intervention clearly described and delivered consistently across the study population? } \\
\hline \multicolumn{4}{|l|}{$\begin{array}{l}\text { 7. Were the outcome measures prespecified, clearly defined, valid, reliable, and assessed consistently across } \\
\text { all study participants? }\end{array}$} \\
\hline \multicolumn{4}{|l|}{ 8. Were the people assessing the outcomes blinded to the participants' exposures/interventions? } \\
\hline \multicolumn{4}{|l|}{ 9. Was the loss to follow-up after baseline $20 \%$ or less? Were those lost to follow-up accounted for in the analysis? } \\
\hline \multicolumn{4}{|l|}{$\begin{array}{l}\text { 10. Did the statistical methods examine changes in outcome measures from before to after the intervention? } \\
\text { Were statistical tests done that provided p values for the pre-to-post changes? }\end{array}$} \\
\hline \multicolumn{4}{|l|}{$\begin{array}{l}\text { 11. Were outcome measures of interest taken multiple times before the intervention and multiple times after } \\
\text { the intervention (i.e., did they use an interrupted time-series design)? }\end{array}$} \\
\hline $\begin{array}{l}\text { 12. If the intervention was conducted at a group level (e.g., a whole hospital, a community, etc.) did the } \\
\text { statistical analysis take into account the use of individual-level data to determine effects at the group level? }\end{array}$ & & & \\
\hline
\end{tabular}

${ }^{*} \mathrm{CD}$, cannot determine; NA, not applicable; NR, not reported

Table 6. Methodological quality assessment of controlled intervention studies.

\begin{tabular}{|l|c|c|c|c|c|c|c|c|c|c|c|c|c|c|c||}
\hline & $\mathbf{1}$ & $\mathbf{2}$ & $\mathbf{3}$ & $\mathbf{4}$ & $\mathbf{5}$ & $\mathbf{6}$ & $\mathbf{7}$ & $\mathbf{8}$ & $\mathbf{9}$ & $\mathbf{1 0}$ & $\mathbf{1 1}$ & $\mathbf{1 2}$ & $\mathbf{1 3}$ & $\mathbf{1 4}$ & Score \\
\hline Shen et al, 2016 (25) & + & $\mathrm{NR}$ & $\mathrm{NR}$ & - & $\mathrm{NR}$ & + & + & + & + & + & + & - & $\mathrm{NA}$ & + & $8 / 14$ \\
\hline Choi et al, 2011 (8) & + & + & + & - & + & + & + & + & + & + & + & + & $\mathrm{NA}$ & + & $12 / 14$ \\
\hline Alcidi et al, 2007 (15) & + & $\mathrm{NR}$ & $\mathrm{NR}$ & - & $\mathrm{NR}$ & + & $\mathrm{NR}$ & $\mathrm{NR}$ & + & + & + & - & $\mathrm{NA}$ & + & $6 / 14$ \\
\hline Ikeuchi et al, 2011 (14) & - & - & - & - & - & + & + & + & + & + & + & - & $\mathrm{NA}$ & + & $7 / 14$ \\
\hline Takahashi et al, 2016 (16) & + & + & + & - & $\mathrm{NR}$ & + & + & + & + & $\mathrm{NR}$ & + & - & $\mathrm{NA}$ & + & $9 / 14$ \\
\hline
\end{tabular}

${ }^{*} \mathrm{CD}$, cannot determine; NA, not applicable; NR, not reported

Table 7. Methodological quality assessment of case and case series studies.

\begin{tabular}{|l|l|l|l|l|l|l|l|l|l|l|l|l|l||}
\hline & $\mathbf{1}$ & $\mathbf{2}$ & $\mathbf{3}$ & $\mathbf{4}$ & $\mathbf{5}$ & $\mathbf{6}$ & $\mathbf{7}$ & $\mathbf{8}$ & $\mathbf{9}$ & $\mathbf{1 0}$ & $\mathbf{1 1}$ & $\mathbf{1 2}$ & Score \\
\hline Clendenen et al, 2015 (20) & + & + & + & + & - & - & + & - & + & - & - & NA & $6 / 12$ \\
\hline Protzman et al, 2014 (12) & + & $\mathrm{NA}$ & + & $\mathrm{NA}$ & - & + & + & - & $\mathrm{NA}$ & $\mathrm{NA}$ & - & $\mathrm{NA}$ & $4 / 12$ \\
\hline Bellini and Barbieri, 2015 (19) & + & + & + & - & - & + & + & - & + & + & - & NA & $7 / 12$ \\
\hline Menzies and Hawkins, 2015(26) & + & $\mathrm{NA}$ & + & $\mathrm{NA}$ & - & + & + & - & $\mathrm{NA}$ & $\mathrm{NA}$ & - & $\mathrm{NA}$ & $4 / 12$ \\
\hline Rojhani et al, 2016 (27) & + & $\mathrm{NA}$ & + & $\mathrm{NA}$ & - & + & + & - & $\mathrm{NA}$ & $\mathrm{NA}$ & - & $\mathrm{NA}$ & $4 / 12$ \\
\hline Kesikburun et al, 2016 (24) & + & + & + & + & - & + & + & - & + & + & - & NA & $8 / 12$ \\
\hline Masala et al, 2014 (23) & + & + & + & + & + & + & + & - & + & + & - & NA & $9 / 12$ \\
\hline Vas et al, 2014 (22) & + & + & + & + & - & + & + & - & + & + & - & NA & $8 / 12$ \\
\hline Fucci et al, 2013 (21) & + & + & + & + & - & + & + & - & + & + & - & NA & $8 / 12$ \\
\hline Akbas et al, 2011 (18) & + & + & + & + & + & + & + & - & + & + & - & NA & $9 / 12$ \\
\hline Karaman et al, 2011 (17) & + & + & + & - & - & + & + & - & - & + & - & NA & $6 / 12$ \\
\hline Sluijter et al, 2008 (25) & + & - & + & NA & - & + & - & - & NA & NA & - & NA & $3 / 12$ \\
\hline
\end{tabular}

${ }^{*} \mathrm{CD}$, cannot determine; NA, not applicable; NR, not reported 
Table 8. USPSTF levels of certainty regarding net benefit (90).

\begin{tabular}{|l|l||}
\hline Level of Certainty & Description \\
\hline High & $\begin{array}{l}\text { The available evidence usually includes consistent results from well-designed, well-conducted studies in representative } \\
\text { primary care populations. These studies assess the effects of the preventive service on health outcomes. This } \\
\text { conclusion is therefore unlikely to be strongly affected by the results of future studies. }\end{array}$ \\
\hline Moderate & $\begin{array}{l}\text { The available evidence is sufficient to determine the effects of the preventive service on health outcomes, but confidence } \\
\text { in the estimate is constrained by such factors as: } \\
\text { The number, size, or quality of individual studies. } \\
\text { Inconsistency of findings across individual studies. } \\
\text { Limited generalizability of findings to routine primary care practice. } \\
\text { Lack of coherence in the chain of evidence. } \\
\text { As more information becomes available, the magnitude or direction of the observed effect could change, and this change } \\
\text { may be large enough to alter the conclusion. }\end{array}$ \\
\hline Low & $\begin{array}{l}\text { The available evidence is insufficient to assess effects on health outcomes. Evidence is insufficient because of: } \\
\text { The limited number or size of studies. } \\
\text { Important flaws in study design or methods. } \\
\text { Inconsistency of findings across individual studies. } \\
\text { Gaps in the chain of evidence. } \\
\text { Findings not generalizable to routine primary care practice. } \\
\text { Lack of information on important health outcomes. } \\
\text { More information may allow estimation of effects on health outcomes. }\end{array}$ \\
\hline
\end{tabular}

tion due to the potential for significant adverse effects, such as GI bleeding, renal impairment, hypersensitivity reactions, and NSAIDS are contraindicated in patients with certain comorbidities, like a history of peptic ulcer disease (50).

For patients unresponsive to oral pharmacologic therapy, intraarticular glucocorticoid injections are the next option in management as they have demonstrated efficacy in numerous clinical trials $(49,51)$. Synthetic glucocorticoid suspensions, such as methylprednisolone or triamcinolone, are the most commonly employed agents for intraarticular injections. A meta-analysis of 28 trials showed that steroid injections of the knee were effective in providing relief from pain at up to 3 weeks post-injection when compared to placebo (51). The symptomatic relief from injections is temporary, often only providing maximal benefit for 2 weeks $(51,52)$. To maintain symptomatic relief, steroid injections are typically repeated at intervals as often as every 3 months; however, the clinical benefits tend to diminish after 2 years of repeated injections (51). Moreover, steroid injections have been associated with numerous long-term complications, such as crystal-induced synovitis, fat necrosis, deterioration of the cartilage, and sepsis (53-56). In short, steroid injections provide immediate, clinically significant relief, but are not a long-term solution for symptomatic control in patients with OA of the knee.

The use of chondroitin and glucosamine, given orally, is another approach, thought to reduce cartilage loss and improve pain, but their efficacy is largely con- troversial (57). Numerous large study trials have shown minimal or no evidence of meaningful benefit, while other trials that suggest benefit have been considerably criticized on their methodologies (57-62).

\section{Invasive Treatment Modalities}

Surgical intervention is reserved for patients with knee OA who have failed conservative treatment approaches. More invasive therapies primarily include arthroscopic debridement and TKA. Even though arthroscopic debridement is used as a means for symptomatic relief of knee OA, it is not supported by the literature. Several randomized trials have demonstrated no significant clinical improvement in patients with knee OA undergoing arthroscopic debridement compared to placebo or compared to physical therapy and medical therapy (63-65). A systematic review of 9 randomized trials compared the benefit of arthroscopic debridement to controls (sham surgery, exercise, medical treatment) and found no clinically significant benefit, but reported a significant occurrence of adverse events like deep venous thrombosis (DVT), pulmonary embolism (PE), infection, and death (65).

Total joint replacement surgery currently remains the definitive treatment for refractory OA of the knee. Patients typically expect to have improved physical utility and decreased pain after undergoing a TKA; however, studies have shown that results are far from perfect. One systematic review showed that $20 \%$ of patients who underwent TKA reported persistent or 
recurrent pain during the following year post-operative (66). A study following patients post-operatively investigated functionality, finding that only $23 \%$ of knees ( N $=223$ ) were rated as functionally excellent; however, clinically $91 \%$ of the knees were rated as excellent (67). The same study found complications in $5 \%$ of patients and revisions were required in $4 \%$. Complications are a feared part of TKA, most notably DVT, PE, and infection. Without prophylaxis, incidence of DVT ranges from $40 \%$ to $88 \%$; however, with prophylaxis, venous thromboembolic events and PE occurred at rates of $1 \%$ and $0.3 \%$, respectively (68). Infection is another grave complication of knee replacement surgery, and even though the incidence is not high (1.6\%), it has been increasing with the growing diabetic population undergoing TKA $(69,70)$. Other adverse events associated with TKA include prosthetic failure, wound problems, arterial injury, and nerve injury. Furthermore, with a major surgery like TKA, recovery time and physical rehabilitation typically lasts at least 8 months, with the most difficult recovery time occurring during the first 3 months (71).

\section{Radiofrequency Ablation}

RFA was first introduced in the 1970s, which evolved as a mechanism to combat chronic pain (7). RFA was originally used to treat trigeminal neuralgia but expanded to address vertebral radiculopathic pain, and is now used in treating conditions ranging from sympathetic derived nerve pain to chronic knee pain (72). RFA of the knee can be performed under fluoroscopic, or other imaging, guidance, with a cannula advanced into the joint towards the area connecting the shaft to the epicondyle. The area is stimulated to identify the nerve position and to ensure that no motor nerves are activated, as evidenced by absence of fasciculations. The RF electrode is then advanced through the cannula to the target area (8). The electrode tip heats up targeted local tissue within a few millimeters to a temperature typically greater than $47^{\circ} \mathrm{C}$ (ranging from $70^{\circ} \mathrm{C}$ to $90^{\circ} \mathrm{C}$ ) for 120 - 130 seconds, generated through an electromagnetic field with a frequency of $250 \mathrm{kHz}(7,8)$.

The mechanism of action of the therapy is that the RF lesion is believed to stop nociceptive (A- $\delta$ and C-fibers) pain input from the periphery to the central nervous system without destroying the motor or sensory (A- $\beta$ ) fibers (7). More specifically, the postulated mechanism of action for clinical benefit of RFA involves the heat generation resulting in thermocoagulation and localized neuronal tissue destruction. These lesions have been shown to demonstrate the characteristics of scar formation, including an acute inflammatory response, cell necrosis, and fibrosis with collagen fiber deposition, occurring over 3 weeks following the procedure. It has been shown that the basal lamina of Schwann cells may be preserved after RFA, which would allow nerve regeneration. The threshold for neuronal tissue destruction has been shown to be $45^{\circ} \mathrm{C}$ in several studies $(7,73,74)$.

The ablative heat is provided via flow of electrical current, generating a well-delineated lesion (73). Additionally, RFA produces a local electrical field, which is thought to promote neuromodulation by inhibition of the excitatory c-fibers $(74,75)$.

\section{Pulsed Radiofrequency Ablation}

Pulsed RFA (PRF) was introduced in 1998 as an alternative to conventional RFA influenced by a study that showed similar patient outcomes with RFA performed at $67^{\circ} \mathrm{C}$ and $40^{\circ} \mathrm{C}(72)$. In this method, the tissue temperature reaches a maximum of $42^{\circ} \mathrm{C}$, which prevents the unwanted adverse effect of irreversible tissue damage $(17,72)$. In PRF, the pulse generator produces pulses with an amplitude of $45 \mathrm{~V}$ lasting 20 milliseconds every 500 milliseconds (twice per second) (76). The generator may modify the parameters in real time to achieve the desired local tissue temperature. Both PRF and RFA have been demonstrated to have similar effects on neuronal conduction, the disruption of which is often reversible $(23,72,76)$. Studies have shown that PRF does not cause irreversible tissue damage or long-lasting structural effects $(77,78)$. The advantages of PRF compared to conventional RFA are that PRF tends to be less neuro-destructive, patients experience less pain with the procedure, and there is minimized tissue destruction. The benefits of PRF are believed to derive from the neuromodulation effects of the local electric field also seen in RFA, but without the tissue damage that is characteristic of the higher localized heat application in RFA. Furthermore, PRF poses less risk for development of deafferentation pain (79). Deafferentation pain is a dreaded, clinically difficult to manage complication that results from injury to nerve bundles, classically seen in patients of orthopedic surgery, limb amputations, spinal cord injuries, and peripheral nerve injuries. These patients experience pain that is severe, unrelenting, and spontaneous in nature in their body distal to the initial nerve insult despite minimal or nonexistent sensitivity to external noxious stimuli. Deafferentation pain probably results from reorganization of the neuronal 
tissue after injury via the pain matrix (80). Essentially, the loss of afferent pain signals to the central nervous system via spinothalamic tracts (deafferentation) causes the pain syndrome. The primary disadvantage of PRF compared to RFA is that PRF produces a shorter duration of relief for patients, requiring the procedure to be performed more frequently to achieve a similar level of pain relief (79).

\section{Cooled Radiofrequency Ablation}

Similar to conventional RFA, water-cooled radiofrequency (CRF) ablation is a novel technology that utilizes thermal ablative mechanisms; however, CRF gives the ability to create a greater local neuronal lesion to increase the changes of effective denervation (27). In CRF, water circulates inside the probe to remove heat, modulating the thermal heat in the tissue to around $60^{\circ} \mathrm{C}$, and alters the overall size, shape, and projections of lesions compared to conventional RFA. It is postulated that the greater sized CRF lesions may reduce the number of technical failures in the setting of a complex and variable neuronal innervation to the knee. The ability to target a greater amount of neuronal tissue is believed to produce long-term pain relief at least to the duration of relief produced by conventional RFA (26).

\section{Discussion}

These 17 studies provide support for the practice of conventional, pulsed, or cooled radiofrequency ablation therapies for the treatment of chronic knee pain in the setting of either OA or post-TKA. Concerns about the application of the findings from these studies arise from many of the common major limitations, including variable or unclear procedural characteristics, lack of head-to-head comparison to other standard treatments, and lack of prolonged follow-up outcomes of treated patients.

\section{Nerve Targets}

Given that the knee has a complex innervation, it is expected to be technically difficult to locate specific nerves and to achieve a desired clinical outcome of pain relief. In 9 out of the 17 studies analyzed $(8,12,17,19,23-$ $25,27,81)$, procedural guidelines specifically targeted the genicular nerve or intraarticular nerves during the RFA procedure. For example, Choi et al (8) and Protzman et al (12) each utilized a conventional RFA approach involving 3 genicular branches: superior lateral (SLGN), superior medial (SMGN), and inferior medial (IMGN). These approaches targeted the connection of the femur shaft to the bilateral femur epicondyles and the connection of the shaft of the tibia to the medial epicondyle, as justified by the close proximity of the nerves to bony landmarks (the metaphyseal to epiphyseal junctions at the epicondyles of the femur and tibia) and a recent recommendation from an anatomic study of cadaver knees (13). As discussed earlier, the anterior aspect of the joint is innervated by branches of the femoral, common peroneal, and saphenous nerves, while the posterior aspect is innervated by branches of the sciatic, tibial, and obturator nerves $(11,12)$. The previously mentioned cadaver knee study (13) also found variable proximal trajectories but consistent distal proximities with the femur and tibia, while suggesting that the inferolateral peroneal nerve branch was inappropriate for RFA due to its close proximity to the common peroneal nerve. Likely for the same reasoning, none of the studies specifically targeted the inferolateral peroneal nerve branch to avoid loss of motor function.

Choi et al (8) investigated conventional RFA of the genicular nerve branches through a randomized, double-blind trial utilizing a sham control procedure, reporting on 17 cases and 18 controls ( 3 patients were lost to follow-up). Outcomes were evaluated using Visual Analog Scale (VAS) scores and Oxford Knee Score (OKS) at one, 4, and 12 weeks post-procedure and showed 59\%, $65 \%$, and $59 \%$ knee pain relief, respectively. Although the follow-up period was brief, the patients experienced significant knee pain relief and functional improvement. Prior to performing the procedures, the authors confirmed the anatomical locations of the genicular nerve branches on 2 cadavers (with supplemental cadaver dissection pictures). Protzman et al (12) also used a conventional RFA approach to target genicular nerve branches to treat a patient experiencing chronic knee pain postTKA. Although the follow-up time was only 3 months, the patient showed significant improvement in knee pain and functionality through the VAS and Knee Injury and Osteoarthritis Outcome Score (KIOOS) ratings. Three other studies $(19,26,27)$ also targeted the genicular nerve with good outcomes in knee pain and functionality, 2 case reports $(26,27)$ and one retrospective case series (19); however, these studies utilized a novel approach, cooled RFA. Bellini and Barbieri (19) analyzed 6 patients who had each received a cooled RFA procedure targeted to the genicular nerves, assessing with VAS scores and the Western Ontario McMaster Universities OA index (WOMAC) at one month, 3 months, 6 months, and 12 months post-procedure. Both scales showed statistically and clinically significant patient improvement out to 
the 12 month follow-up time point. Rojhani et al (27) discussed the case of a bilateral knee OA patient who received cooled RFA targeted to the bilateral superior lateral, superior medial, and inferior medial geniculate nerves. Up to 3 months after the procedure, the patient reported clinical and functional improvement as measured by the Numerical Rating Scale (NRS), Medical Outcomes Study 36-Item Short-Form Health Survey (SF-36), and the WOMAC. Menzies and Hawkins (26) reported on a patient who suffered chronic knee pain despite bilateral TKA and underwent cooled RFA of the bilateral superior lateral, superior medial, and inferior medial genicular nerves. As assessed with the OKS, the patient demonstrated marked clinical and functional improvement up to 6 months post-procedure in the right knee and 9 months post-procedure in the left knee. Of these 5 studies targeting the genicular nerve, only Choi et al (8) was a controlled intervention study and scored $12 / 14$ by our scale, suggesting it was well designed. The remaining 4 studies were case or case series studies, scoring 4/12 (Protzman et al [12]), 7/12 (Bellini and Barbieri [19]), 4/12 (Menzies and Hawkins [26]), and 4/12 (Rojhani et al [27]). These studies were often limited by small sample sizes and a limited amount of pre-intervention data reporting. Based on the evidence analyzed, targeting of the genicular nerve is likely a low level of certainty; however, Choi et al (8) suggests that this nerve target could be promising in future, larger randomized controlled trials.

Three studies $(17,23,25)$ utilized PRF via an intraarticular approach under fluoroscopic guidance and applied the PRF probe for either 5, 10, or 15 minutes. The patients in these studies experienced significant relief of pain (VAS or NRS) and improvement in quality of life (WOMAC score) at all follow-ups from one month to 10 months. The prospective case series of 40 patients in Masala et al (23) showed 73\% improvement after only one week from assessment by outcome scores (VAS, WOMAC, painDETECT), which is quickest and greatest improvement shown by any studies. None of these 3 studies were controlled trials, and scored 6/12 (Karaman et al [17]), 9/12 (Masala et al [23]), and 3/12 (Sluijter et al [25]). While well-designed overall, there is a low level of certainty for the nerve targets emphasized in these trials.

A non-randomized, prospective controlled open label trial by lkeuchi et al (14) with 35 patients targeted the medial retinacular nerve and the infrapatellar branch of the saphenous nerve (IPSN) with a 2-treatment conventional RFA for anteromedial knee OA pain. The study provided explicit description of the technical details for the anteromedial approach and delineated anatomical landmarks with a diagram, but the study procedure used a palpation-based technique rather than fluoroscopic or ultrasound guidance. The results showed that RFA significantly improved knee pain at all follow-up time points to 12 weeks compared to the control group of nerve block only. The RF group, however, averaged lower on the WOMAC total score (indicating less disability) throughout the treatment cycle, including at baseline, with no significant difference found between the groups. The percentage of responders in the RFA group according to VAS score was approximately $30 \%$ at 12 weeks and less than $10 \%$ at the 6-month follow-up. The paper noted a concern that there was no proof that the target nerves were truly responsible for the patients' clinical symptoms. Despite this concern, their rationale in choosing the nerve targets for RFA outlines that these nerves innervate the anteromedial aspect of intraarticular structures, which is thought to be largely responsible for knee pain in OA $(14,81)$. Overall, the study by Ikeuchi et al (14) contains concerns about the efficacy of the approach to the nerve targets using a palpation-based technique and highlights the possibility of a placebo effect for the procedure versus the control nerve block procedure. The retrospective case series of 2 patients by Clendenen et al (20) also targeted the infrapatellar branch of the saphenous nerve (IPSN) using conventional RFA. In this series, 2 post-TKA patients with recurrent symptoms despite hydrodissection of the nerve with local steroid injection were then treated by RFA of the IPSN under ultrasound guidance. After the RFA ( $2-4$ sessions at $80^{\circ} \mathrm{C}$ for 90 seconds each), $4 \mathrm{mg}$ dexamethasone (more potent than the $20 \mathrm{mg}$ of methylprednisolone used in the first treatment) was injected before removing the RFA needle. At 12-months post-procedure, one patient had complete resolution (VAS score 0 ) of knee pain while the other still experienced symptoms. The confounding variable of steroid injection should not be overlooked in these outcomes of Clendenen et al (20). Furthermore, the authors noted that their experience with RFA was very limited. Ikeuchi et al (14) was a controlled trial that scored a 7/14 while Clendenen et al (20) was a case series that scored a $6 / 12$. While these results suggest targeting the infrapatellar branch of the saphenous nerve may not be the most effective RFA target, there is a low level of certainty in this recommendation.

Three case series studies $(18,21,22)$ used PRF targeted to large nerves, such as the femoral, saphenous, tibial, and sciatic nerves. Akbas et al (18) studied 115 
patients in a retrospective case series targeting the saphenous nerve, and thus targeting the IPSN at the tibial tuberosity. Benefit from the PRF was shown on the tenth day for $100 \%$ of patients with VAS decreased by $>83 \%$ ( $>81 \%$ total reduction score at 6 months). All patients in this study, however, continued their medication regimen for pain control during the trial; the study did not clarify which medications the patients were taking for analgesia. The prospective case series of 25 patients (OA, post-TKA, or posttraumatic knee pain) by Fucci et al (21) targeted the sciatic nerve with PRF under ultrasound guidance. The sciatic nerve was approached posterior just prior to the division into tibial and peroneal nerves. Patients improved an average of only 27/100 millimeters on the VAS scale 4-weeks post-procedure, although this was statistically significant. The follow-up period was limited to 4 weeks, one of the shortest in all the studies. Only 5 patients had achieved a $20 \%$ improvement in walking distance. Most surprisingly, out of the 47 knee PRF procedures performed, 10 failed (same pre and post VAS scores), which meant that these patients experienced a total absence of pain relief. Although the rationale in targeting the sciatic nerve was to increase efficacy by covering a larger intra-capsular sensory area, the study failed to match the efficacy of Choi et al (8), which averaged 45/100 millimeters VAS score improvement by ablating the genicular nerves. Vas et al (22) looked at 10 patients in a prospective case series treating their knee pain by targeting the entire nerve supply of the knee with ultrasound-guided PRF. Both sensory and motor nerves, the femoral, saphenous, tibial, common peroneal nerves and peripatellar, subsartorial, and popliteal plexuses, were targeted in attempt to address nociception and stiffness leading to peripheral and central sensitization in knee OA. All patients showed significant improvement in outcome sores - NRS, painDetect, and WOMAC - at 3 and 6 months post-PRF. $X$-ray finding post-PRF showed a reduction of inflammation and swelling, which the authors reasoned was a result of decreased joint pain and reflex spasms. This premise is thought to involve reduction of afferent nociceptive input to the neuraxis in conjunction with muscle relaxation by reduction of efferent outflow of motor response to pain and inflammation. Patients reported muscle relaxation and decreased stiffness, which enabled them to optimize physical therapy. The publication detailed the pre-procedural pain profiles of the knees bilaterally, limits of activities, x-ray findings, and specific conservative treatments taken. Compre- hensive descriptions of the targeted nerve innervation of the knee with associated diagrams and ultrasound snapshots from the study were provided, which could prove useful for reproduction of the procedure. With the ultrasound-guided approach, the time to ablate all nerves for one knee typically took only 45 - 50 minutes. Additionally, post-procedure patients received customized physiotherapy programs. Albeit a small study of only 10 patients, Vas et al (22) showed promising results in extensive PRF of the knee through a comprehensive publication. All 3 of these studies were well-designed, Akbas et al scoring 9/12 (18), Fucci et al scoring 8/12 (21), and Vas et al scoring 8/12 (22). Despite being welldesigned, all 3 trials were case series and provide a low level of certainty that targeting the larger nerves of the knee is not an effective RFA approach.

Two studies $(6,15)$ did not sufficiently specify the nerve target for RFA. In a randomized controlled trial of 54 patients, Shen et al (6) investigated RFA plus injection of platelet-rich plasma and sodium hyaluronate compared to injection alone. The authors described their approach as "we applied RFTC to the peripheral nerve of the knee joint." The technical details of the procedure were unclear, and the rationale in comparing RFA with injection of platelet-rich plasma and hyaluronate was not discussed. Patient outcomes showed significant improvement in VAS scores and QoL assessments at the short 3-month follow-up time when compared to controls. The strongest possible conclusion from this study is that RFA with injection has a clinical advantage to injection alone; unfortunately, this study cannot be reproduced due to the lack of procedural details. Another randomized controlled trial by Alcidi et al (15) investigated the efficacy of low power RFA versus transcutaneous electrical nerve stimulation (TENS) in a study of 40 patients. The patients received treatment sessions of 20 minutes each for 5 consecutive days. The study, however, did not clarify the targeted nerve of the knee, or if there were any specifically targeted nerves. The trial outcomes from VAS score and Lequesne's index, which measures disability, showed significant improvement of knee pain and function in the case group receiving RFA. With the absence of a placebo control group, the only conclusion that can be drawn from this study is that low frequency RFA may be have a clinically significant edge over TENS for treatment of knee OA. Without a specific nerve target, it is difficult to make a concrete statement about the efficacy of RFA from these 2 trials, despite both being controlled trials that scored 8/14 (Shen et al [6]) and 6/14 (Alcidi et al [15]). 


\section{Imaging Modality}

For imaging modalities in the reviewed studies, fluoroscopy, ultrasonography, or a combination of both were used during the procedures to visualize anatomical structures. Seven studies performed the procedure under fluoroscopic guidance, including 3 CRF studies $(19,26,27), 3 \operatorname{PRF}(17,23,25)$, and one conventional approach (8). Four publications utilized ultrasound guidance during the ablation procedure, 3 of which utilized PRF $(21,22,24)$ while Clendenen et al (20) utilized conventional RFA and Protzman et al (12) applied both methodologies during a conventional RFA procedure. Meanwhile, the other 5 studies $(6,14-16,18)$ did not use any imaging, but simply used physical exam landmarks or failed to clearly specify whether imaging was or was not used. Kesikburun et al (24) performed ultrasound-guided PRF targeting the genicular nerves on 9 patients. Ultrasound-guided genicular nerve block was performed in the initial 49 patients of the study, and those patients who had initial pain relief that faded over time were candidates for ultrasound-guided PRF genicular nerve ablation. The superior medial and inferior medial genicular nerves were targeted by applying the probe at $42^{\circ} \mathrm{C}$ for 4 minutes at each target. The authors' reasoning for only targeting 2 of the genicular nerve branches was the thought that these are the only 2 nerves clinically relevant in pain from knee OA of the medial compartment. This study described the ultrasound approach and technique in great detail with several figures for clarity of the neurovascular bundles at the anatomical landmarks, which may be exceptionally valuable for physicians desiring to utilize the same technique with their patients. A significant reduction in VAS and WOMAC scores was detected at one, 4 , and 12 week follow-up points in all the patients who underwent ultrasound-guided PRF.

Yasar et al (82) employed the anatomical approach proven to be accurate by a recent cadaver study from 2015 investigating ultrasound-guided genicular nerve blocks. Furthermore, Fucci et al (21) and Vas et al (22) applied ultrasound-guided PRF, finding significant improvement in their patient's reported knee pain and functionality at follow-up. Protzman et al (12) applied fluoroscopy to identify bony landmarks with subsequent ultrasound to find the genicular nerve branches in patients with chronic knee pain post-TKA. Since ultrasound-guided approaches are inexpensive, easily reproduced, and are without exposure to ionizing radiation, this imaging modality has advantages over traditional fluoroscopy. Thus, ultrasonography has recently become more prevalent in pain management and peripheral nerve blocks (83).

Of the 5 studies analyzed that were controlled intervention studies, 4 did not utilize any imaging modality (Shen et al [6], Alcidi et al [15], Ikeuchi et al [14], and Takahashi et al [16]). While the studies that report using imaging guidance, whether fluoroscopy or ultrasound, tend to report positive outcomes, it is difficult to make a statement about the necessity of imaging in RFA.

\section{Comparing Methodologies}

These currently published studies make it difficult to compare the effectiveness of radiofrequency ablation between the 3 different procedure types. All of the published studies $(6,8,12,14-27)$ in this review report positive patient outcomes regardless of the type of ablation procedure used. Clendenen et al (20) were the only authors to report a result of less than significant pain relief following a conventional RFA procedure, which occurred in one of the 2 patients receiving ablation in the study. The details of this patient are not discussed, other than a modest VAS improvement from $7 / 10$ at baseline to $4 / 10$ at a 12-month follow-up.

There is also a lack of consistent evidence regarding the adverse events experienced by the patients receiving procedures in these studies. Of the 6 studies analyzing conventional RFA $(6,8,12,14,15,20), 4$ studies do not mention any adverse event reporting $(6,12,15,20)$, one study mentions that no adverse events were reported (8), and one study (with an $\mathrm{N}=35$ ) reports that $67 \%$ of patients experienced minor subcutaneous bleeding at the injection site and $78 \%$ reported prolonged hypoesthesia (14). Of the 4 studies analyzing $\operatorname{CRF}(16,19,26,27)$, 3 studies mentioned that there were no adverse events reported $(19,26,27)$ and one study did not mention any adverse event reporting (16). Of the 7 studies analyzing PRF (17,18,21-25), 6 studies mentioned that no adverse events were reported $(17,18,21-24)$ and one study did not mention any adverse event reporting (25). While the only reported adverse events in these studies occurred following conventional RFA procedures (14), the low severity of these adverse events suggests that this may not be a strong reason to definitively separate the procedure types.

Unfortunately, it is difficult to make a specific statement about CRF at this time. While a commercially available CRF prove is available, many studies utilize a cooled procedure with conventional probes. Until more research is done comparing a cooled procedure using a conventional probe versus the marketed cooled probe, 
a conclusion about the effectiveness of CRF cannot be made at this time.

Overall, all 3 RFA procedures appear to have similar safety and benefit profiles. While the body of evidence as a whole may be enough to support a moderate level of certainty for the benefits of RFA for treatment of knee pain, the wide variety of procedures, nerve targeting, imaging modality, and patient follow-up suggests that there is a low level of certainty that there is a benefit from any specific RFA procedure.

\section{Future Directions}

Currently, a randomized controlled open label clinical trial of CRF sponsored by Halyard Health (Nerve Ablation by Cooled Radiofrequency Compared to Corticosteroid Injection for Management of Knee Pain - NCT02343003) (84) is underway at 11 institutions with an estimated enrollment of 144 patients. The study plans to evaluate outcomes using NRS, OKS, and WOMAC scoring systems at 6 and 12 month follow-up time points. The study should substantiate the small body of literature of case reports and retrospective case series on CRF.

To determine the role of imaging modalities, there is a need for larger trials to assess which modalities carry advantages for reducing technical failures and reducing risk of adverse events. The current body of evidence suggests that ultrasound-guided approaches are safe and effective, and this evidence could prompt the adoption of this imaging modality in most procedures performed in clinical settings.

It is also important to note that many of the studies analyzed in this review do not discuss the impact of opioid therapy on enrolled patients. All of the studies provide a rationale to support ablation as a clinical option following failure of conventional drug treatment (steroids and analgesics) and/or knee replacement, but there is no evidence provided for the impact of ablation treatment on patients who rely on opioid therapy for pain relief. The promise of ablation therapy as a method of opioid-sparing pain control should be a target for future research.

\section{Conclusion}

Even though the literature regarding the efficacy of RFA is limited, there is strong evidence that supports RFA in treating chronic knee pain due to OA or post-TKA pain syndrome. Two of the randomized clinical trials should be taken cautiously $(6,15)$, while other studies, such as Choi et al (8), Protzman et al (12), Vas et al (22), Sluijter et al (25), and Ikeuchi et al (14), show encouraging results and delineate several solid approaches in radiofrequency therapy. Targeting the genicular nerve branches has had a profound clinical benefit $(8,12,19,24,26,27)$, as well as treating the entire nerve and motor supply to the knee, although this approach can be excessive and less cost-effective (22).

Patients with moderate to severe OA of the knee who are refractory to conservative treatment modalities are left with TKA as a terminal treatment option. Even with an established treatment such as TKA with mostly satisfactory results, there still remains a substantial percentage, up to $7 \%$, of patients who experience debilitating knee pain after TKA $(26,66,85)$. Thus, it is imperative to educate patients and physicians about novel promising treatment alternatives, such as RFA and $C R F$, to treat chronic pain patients who may have limited options.

\section{References}

1. Garstang SV, Stitik TP. Osteoarthritis: Epidemiology, risk factors, and pathophysiology. Am J Phys Med Rehabil 2006; $85: S_{2}-S_{11}$.

2. Dillon CF, Rasch EK, Gu Q, Hirsch R. Prevalence of knee osteoarthritis in the United States: Arthritis data from the Third National Health and Nutrition Examination Survey 1991-94. J Rheumatol 2006; 33:2271-2279.

3. Guccione AA, Felson DT, Anderson JJ, Anthony JM, Zhang Y, Wilson PW, Kel-
ly-Hayes M, Wolf PA, Kreger BE, Kannel WB. The effects of specific medical conditions on the functional limitations of elders in the Framingham Study. Am J Public Health 1994; 84:351-358.

4. Michael JW, Schluter-Brust KU, Eysel P. The epidemiology, etiology, diagnosis, and treatment of osteoarthritis of the knee. Dtsch Arztebl Int 2010; 107:152-162.

5. McHugh GA, Luker KA, Campbell M, Kay PR, Silman AJ. Pain, physical functioning and quality of life of individuals awaiting total joint replacement: A longitudinal study. J Eval Clin Pract 2008; 14:19-26.

6. Shen WS, Xu XQ, Zhai NN, Zhou ZS, Shao J, Yu YH. Radiofrequency thermocoagulation in relieving refractory pain of knee osteoarthritis. Am J Ther 2016. doi: 10.5812/aapm.39696.

7. Kapural L, Mekhail N. Radiofrequency ablation for chronic pain control. Curr Pain Headache Rep 2001; 5:517-525.

8. Choi WJ, Hwang SJ, Song JG, Leem JG, Kang YU, Park PH, Shin JW. Radiofre- 
quency treatment relieves chronic knee osteoarthritis pain: A double-blind randomized controlled trial. Pain 2011; 152:481-487.

9. Kim SY, Le PU, Kosharskyy B, Kaye AD, Shaparin N, Downie SA. Is genicular nerve radiofrequency ablation safe? A literature review and anatomical study. Pain Physician 2016; 19:E697-E705.

10. Hirasawa Y, Okajima S, Ohta M, Tokioka T. Nerve distribution to the human knee joint: Anatomical and immunohistochemical study. Int Orthop 2000; 24:1-4.

11. Kennedy JC, Alexander IJ, Hayes KC. Nerve supply of the human knee and its functional importance. Am J Sports Med 1982; 10:329-335.

12. Protzman NM, Gyi J, Malhotra AD, Kooch JE. Examining the feasibility of radiofrequency treatment for chronic knee pain after total knee arthroplasty. PM R 2014; 6:373-376.

13. Franco CD, Buvanendran A, Petersohn JD, Menzies RD, Menzies LP. Innervation of the anterior capsule of the human knee: Implications for radiofrequency ablation. Reg Anesth Pain Med 2015; 40:363-368.

14. Ikeuchi M, Ushida T, Izumi M, Tani T. Percutaneous radiofrequency treatment for refractory anteromedial pain of osteoarthritic knees. Pain Med 2011; 12:546-551.

15. Alcidi L, Beneforti E, Maresca M, Santosuosso U, Zoppi M. Low power radiofrequency electromagnetic radiation for the treatment of pain due to osteoarthritis of the knee. Reumatismo 2007; 59:140-145.

16. Takahashi K, Hashimoto S, Kurosaki H, Kato K, Majima T, Shindo Y, Watanabe $\mathrm{H}$, Mochizuki $Y$, Takai S. A pilot study comparing the efficacy of radiofrequency and microwave diathermy in combination with intra-articular injection of hyaluronic acid in knee osteoarthritis. ] Phys Ther Sci 2016; 28:525-529.

17. Karaman H, Tufek A, Kavak GO, Yildirim ZB, Uysal E, Celik F, Kaya S. Intra-articularly applied pulsed radiofrequency can reduce chronic knee pain in patients with osteoarthritis. J Chin Med Assoc 2011; 74:336-340.

18. Akbas M, Luleci N, Dere K, Luleci E, Ozdemir U, Toman H. Efficacy of pulsed radiofrequency treatment on the saphenous nerve in patients with chronic knee pain. J Back Musculoskelet Rehabil 2011; 24:77-82.
19. Bellini M, Barbieri M. Cooled radiofrequency system relieves chronic knee osteoarthritis pain: The first case-series. Anaesthesiol Intensive Ther 2015; 47:30-33.

20. Clendenen S, Greengrass R, Whalen J, O'Connor MI. Infrapatellar saphenous neuralgia after TKA can be improved with ultrasound-guided local treatments. Clin Orthop Relat Res 2015; 473:119-125.

21. Fucci R, Pascual-Ramirez J, MartinezMarcos A, Mantecon JM. Ultrasoundguided sciatic nerve pulsed radiofrequency for chronic knee pain treatment: A novel approach. J Anesth 2013; 27:935-938.

22. Vas L, Pai R, Khandagale N, Pattnaik M. Pulsed radiofrequency of the composite nerve supply to the knee joint as a new technique for relieving osteoarthritic pain: A preliminary report. Pain Physician 2014; 17:493-506.

23. Masala S, Fiori R, Raguso M, Morini M, Calabria E, Simonetti G. Pulsedose radiofrequency for knee osteoartrithis. Cardiovasc Intervent Radiol 2014; 37:482-487.

24. Kesikburun S, Yasar E, Uran A, Adiguzel E, Yilmaz B. Ultrasound-guided genicular nerve pulsed radiofrequency Treatment for painful knee osteoarthritis: A preliminary report. Pain Physician 2016; 19:E751-E759.

25. Sluijter ME, Teixeira A, Serra V, Balogh $S$, Schianchi P. Intra-articular application of pulsed radiofrequency for arthrogenic pain -- report of six cases. Pain Pract 2008; 8:57-61.

26. Menzies RD, Hawkins JK. Analgesia and improved performance in a patient treated by cooled radiofrequency for pain and dysfunction postbilateral total knee replacement. Pain Pract 2015; 15:E54-E58.

27. Rojhani S, Qureshi Z, Chhatre A. Watercooled radiofrequency provides pain relief, decreases disability, and improves quality of life in chronic knee osteoarthritis. Am J Phys Med Rehabil 2017; 96:e5-e8.

28. Felson DT, Anderson J, Naimark A, Walker AM, Meenan RF. Obesity and knee osteoarthritis. The Framingham Study. Ann Intern Med 1988; 109:18-24.

29. Felson DT. The epidemiology of knee osteoarthritis: Results from the Framingham Osteoarthritis Study. Semin Arthritis Rheum 1990; 20:42-50.

30. Felson DT, Zhang Y, Anthony JM, Nai- mark A, Anderson JJ. Weight loss reduces the risk for symptomatic knee osteoarthritis in women. The Framingham Study. Ann Intern Med 1992; 116:535-539.

31. Messier SP, Mihalko SL, Legault C, Miller GD, Nicklas BJ, DeVita P, Beavers DP, Hunter DJ, Lyles MF, Eckstein F, Williamson JD, Carr JJ, Guermazi A, Loeser RF. Effects of intensive diet and exercise on knee joint loads, inflammation, and clinical outcomes among overweight and obese adults with knee osteoarthritis: The IDEA randomized clinical trial. JAMA 2013; 310:1263-1273.

32. Messier SP, Loeser RF, Miller GD, Morgan TM, Rejeski WJ, Sevick MA, Ettinger WH Jr, Pahor M, Williamson JD. Exercise and dietary weight loss in overweight and obese older adults with knee osteoarthritis: The arthritis, diet, and activity promotion trial. Arthritis Rheum 2004; 50:1501-1510.

33. Aguiar GC, Do Nascimento MR, De Miranda AS, Rocha NP, Teixeira AL, Scalzo PL. Effects of an exercise therapy protocol on inflammatory markers, perception of pain, and physical performance in individuals with knee osteoarthritis. Rheumatol Int 2015; 35:525-531.

34. Abbott JH, Robertson MC, Chapple C, Pinto D, Wright AA, Leon de la Barra S, Baxter GD, Theis JC, Campbell AJ; MOA Trial team. Manual therapy, exercise therapy, or both, in addition to usual care, for osteoarthritis of the hip or knee: A randomized controlled trial. 1: Clinical effectiveness. Osteoarthritis Cartilage 2013; 21:525-534.

35. Bennell KL, Hinman RS, Metcalf BR, Buchbinder R, McConnell J, McColl G, Green S, Crossley KM. Efficacy of physiotherapy management of knee joint osteoarthritis: A randomised, double blind, placebo controlled trial. Ann Rheum Dis 2005; 64:906-912.

36. Fransen M, Crosbie J, Edmonds J. Physical therapy is effective for patients with osteoarthritis of the knee: A randomized controlled clinical trial. J Rheumatol 2001; 28:156-164.

37. Deyle GD, Henderson NE, Matekel RL, Ryder MG, Garber MB, Allison SC. Effectiveness of manual physical therapy and exercise in osteoarthritis of the knee. A randomized, controlled trial. Ann Intern Med 2000; 132:173-181.

38. Fransen M, McConnell S, Harmer AR, Van der Esch M, Simic M, Bennell $\mathrm{KL}$. Exercise for osteoarthritis of the knee. Cochrane Database Syst Rev 2015; 
1:CDoo4376.

39. Fitzgerald GK, Fritz JM, Childs JD, Brennan GP, Talisa V, Gil AB, Neilson BD, Abbott $\mathrm{JH}$. Exercise, manual therapy, and use of booster sessions in physical therapy for knee osteoarthritis: A multi-center, factorial randomized clinical trial. Osteoarthritis Cartilage 2016; 24:1340-1349.

40. Wang $\mathrm{C}$, Schmid $\mathrm{CH}$, Iversen $\mathrm{MD}$, Harvey WF, Fielding RA, Driban JB, Price LL, Wong JB, Reid KF, Rones R, McAlindon T. Comparative effectiveness of tai chi versus physical therapy for knee osteoarthritis: A randomized trial. Ann Intern Med 2016; 165:77-86.

41. Thorstensson CA, Roos EM, Petersson IF, Ekdahl C. Six-week high-intensity exercise program for middle-aged patients with knee osteoarthritis: A randomized controlled trial [ISRCTN20244858]. BMC Musculoskelet Disord 2005; 6:1-10.

42. Bartels EM, Juhl CB, Christensen $\mathrm{R}, \mathrm{Ha}$ gen KB, Danneskiold-Samsoe B, Dagfinrud $\mathrm{H}$, Lund $\mathrm{H}$. Aquatic exercise for the treatment of knee and hip osteoarthritis. Cochrane Database Syst Rev 2016; 3:CDoo5523.

43. McNeal RL. Aquatic therapy for patients with rheumatic disease. Rheum Dis Clin North Am 1990; 16:915-929.

44. Chamberlain MA, Care G, Harfield B. Physiotherapy in osteoarthrosis of the knees. A controlled trial of hospital versus home exercises. Int Rehabil Med 1982; 4:101-106.

45. Blair SN, Kohl HW 3rd, Paffenbarger RS Jr, Clark DG, Cooper KH, Gibbons LW. Physical fitness and all-cause mortality: A prospective study of healthy men and women. JAMA 1989; 262:2395-2401.

46. Hochberg MC, Altman RD, April KT, Benkhalti M, Guyatt G, McGowan J, Towheed T, Welch V, Wells G, Tugwell P; American College of Rheumatology. American College of Rheumatology 2012 recommendations for the use of nonpharmacologic and pharmacologic therapies in osteoarthritis of the hand, hip, and knee. Arthritis Care Res (Hoboken) 2012; 64:465-474.

47. da Costa BR, Reichenbach S, Keller N, Nartley L, Wandel S, Juni P, Trelle S. Effectiveness of non-steroidal antiinflammatory drugs for the treatment of pain in knee and hip osteoarthritis: A network meta-analysis. Lancet 2016; 387:2093-2105.

48. Bjordal JM, Klovning A, Ljunggren AE,
Slørdal L. Short-term efficacy of pharmacotherapeutic interventions in osteoarthritic knee pain: A meta-analysis of randomised placebo-controlled trials. Eur J Pain 2007; 11:125-138.

49. Bannuru RR, Schmid CH, Kent DM, Vaysbrot EE, Wong JB, McAlindon TE. Comparative effectiveness of pharmacologic interventions for knee osteoarthritis: A systematic review and network meta-analysis. Ann Intern Med 2015; 162:46-54.

50. Bozimowski G. A review of nonsteroidal anti-inflammatory drugs. AANA J. 2015; 83:425-433.

51. Bellamy N, Campbell J, Robinson V, Gee T, Bourne R, Wells G. Intraarticular corticosteroid for treatment of osteoarthritis of the knee. Cochrane Database Syst Rev 2006; 19:CDoo5328.

52. Bjordal JM, Klovning A, Ljunggren AE, Slordal L. Short-term efficacy of pharmacotherapeutic interventions in osteoarthritic knee pain: A meta-analysis of randomised placebo-controlled trials. Eur J Pain 2007; 11:125-138.

53. McDonough AL. Effects of corticosteroids on articular cartilage: A review of the literature. Phys Ther 1982; 62:835-839.

54. Rozental TD, Sculco TP. Intra-articular corticosteroids: An updated overview. Am J Orthop (Belle Mead NJ) 2000; 29:18-23.

55. Bellamy N, Campbell J, Robinson V, Gee T, Bourne R, Wells G. Intraarticular corticosteroid for treatment of osteoarthritis of the knee. Cochrane Database Syst Rev 2005; 19:CDoo5328.

56. Seror $P$, Pluvinage $P$, d'Andre FL, Benamou P, Attuil G. Frequency of sepsis after local corticosteroid injection (an inquiry on 1160000 injections in rheumatological private practice in France). Rheumatology (Oxford) 1999; 38:1272-1274.

57. Clegg DO, Reda DJ, Harris CL, Klein MA, O'Dell JR, Hooper MM, Bradley JD, Bingham $\mathrm{CO}$ 3rd, Weisman $\mathrm{MH}$, Jackson CG, Lane NE, Cush JJ, Moreland LW, Schumacher HR Jr, Oddis CV, Wolfe F, Molitor JA, Yocum DE, Schnitzer TJ, Furst DE, Sawitzke AD, Shi $\mathrm{H}$, Brandt KD, Moskowitz RW, Williams HJ. Glucosamine, chondroitin sulfate, and the two in combination for painful knee osteoarthritis. N Engl J Med 2006; 354:795-808.

58. Sawitzke AD, Shi H, Finco MF, Dunlop DD, Harris CL, Singer NG, Bradley JD, Silver D, Jackson CG, Lane NE, Oddis
CV, Wolfe F, Lisse J, Furst DE, Bingham CO, Reda DJ, Moskowitz RW, Williams HJ, Clegg DO. Clinical efficacy and safety of glucosamine, chondroitin sulphate, their combination, celecoxib or placebo taken to treat osteoarthritis of the knee: 2-year results from GAIT. Ann Rheum Dis 2010; 69:1459-1464.

59. Towheed TE, Maxwell L, Anastassiades TP, Shea B, Houpt J, Robinson V, Hochberg MC, Wells G. Glucosamine therapy for treating osteoarthritis. Cochrane Database Syst Rev 2005; 18:CDo02946.

6o. Reginster JY, Deroisy R, Rovati LC, Lee RL, Lejeune E, Bruyere O, Giacovelli G, Henrotin Y, Dacre JE, Gossett C. Longterm effects of glucosamine sulphate on osteoarthritis progression: A randomised, placebo-controlled clinical trial. Lancet 2001; 357:251-256.

61. Martel-Pelletier J, Roubille C, Abram F, Hochberg MC, Dorais M, Delorme P, Raynauld JP, Pelletier JP. First-line analysis of the effects of treatment on progression of structural changes in knee osteoarthritis over 24 months: Data from the osteoarthritis initiative progression cohort. Ann Rheum Dis 2015; 74:547-556.

62. Wu D, Huang Y, Gu Y, Fan W. Efficacies of different preparations of glucosamine for the treatment of osteoarthritis: A meta-analysis of randomised, double-blind, placebo-controlled trials. Int J Clin Pract 2013; 67:585-594.

63. Moseley JB, O'Malley K, Petersen NJ, Menke TJ, Brody BA, Kuykendall DH, Hollingsworth JC, Ashton CM, Wray NP. A controlled trial of arthroscopic surgery for osteoarthritis of the knee. $N$ Eng J Med 2002; 347:81-88.

64. Kirkley A, Birmingham TB, Litchfield RB, Giffin JR, Willits KR, Wong CJ, Feagan BG, Donner A, Griffin SH, D'Ascanio LM, Pope JE, Fowler PJ. A randomized trial of arthroscopic surgery for osteoarthritis of the knee. N Eng J Med 2008; 359:1097-1107.

65. Thorlund JB, Juhl CB, Roos EM, Lohmander LS. Arthroscopic surgery for degenerative knee: Systematic review and meta-analysis of benefits and harms. $\mathrm{Br}$ J Sports Med 2015; 49:1229-1235.

66. Wylde V, Hewlett S, Learmonth ID, Dieppe P. Persistent pain after joint replacement: Prevalence, sensory qualities, and postoperative determinants. Pain 2011; 152:566-572.

67. Gill GS, Joshi AB. Long-term results of cemented, posterior cruciate liga- 
ment-retaining total knee arthroplasty in osteoarthritis. Am J Knee Surg 2001; 14:209-214.

68. Januel JM, Chen G, Ruffieux C, Quan $H$, Douketis JD, Crowther MA, Colin C, Ghail WA, Burnand B, IMECCHI Group. Symptomatic in-hospital deep vein thrombosis and pulmonary embolism following hip and knee arthroplasty among patients receiving recommended prophylaxis: A systematic review. JAMA 2012; 307:294-303.

69. Wilson MG, Kelley K, Thornhill TS. Infection as a complication of total kneereplacement arthroplasty. Risk factors and treatment in sixty-seven cases. J Bone Joint Surg Am 1990; 72:878-883.

70. Yang K, Yeo SJ, Lee BP, Lo NN. Total knee arthroplasty in diabetic patients: A study of 109 consecutive cases. J Arthroplasty 2001; 16:102-106.

71. Kennedy DM, Stratford PW, Riddle DL, Hanna SE, Gollish JD. Assessing recovery and establishing prognosis following total knee arthroplasty. Phys Ther 2008; 88:22-32.

72. Bogduk N. Pulsed radiofrequency. Pain Med 2006; 7:396-407.

73. Cosman ER, Jr., Cosman ER, Sr. Electric and thermal field effects in tissue around radiofrequency electrodes. Pain Med 2005; 6:405-424.

74. Smith HP, McWhorter JM, Challa VR. Radiofrequency neurolysis in a clinical model. Neuropathological correlation. J Neurosurg 1981; 55:246-253.

75. Slappendel R, Crul BJ, Braak GJ, Geurts JW, Booij LH, Voerman VF, de Boo T. The efficacy of radiofrequency lesioning of the cervical spinal dorsal root ganglion in a double blinded randomized study: No difference between 40 degrees $C$ and 67 degrees $C$ treatments.
Pain 1997; 73:159-163.

76. Cahana A, Van Zundert J, Macrea L, van Kleef M, Sluijter M. Pulsed radiofrequency: Current clinical and biological literature available. Pain Med 2006; 7:411-423.

77. Kvarstein G. Pulsed radiofrequency -time for a clinical pause and more science. Scand J Pain 2012; 3:124-126.

78. Tun K, Cemil B, Gurcay AG, Kaptanoglu E, Sargon MF, Tekdemir I, Comert A, Kanpolat Y. Ultrastructural evaluation of pulsed radiofrequency and conventional radiofrequency lesions in rat sciatic nerve. Surg Neurol 2009; 72:496-500.

79. Pangarkar S, Miedema ML. Pulsed versus conventional radio frequency ablation for lumbar facet joint dysfunction. Curr Phys Med Rehabil Rep 2014; 2:61-65.

8o. Hanakawa T. Neural mechanisms underlying deafferentation pain: A hypothesis from a neuroimaging perspective. J Orthop Sci 2012; 17:331-335.

81. Creamer P, Hunt M, Dieppe P. Pain mechanisms in osteoarthritis of the knee: Effect of intraarticular anesthetic. J Rheumatol 1996; 23:1031-1036.

82. Yasar E, Kesikburun S, Kilic C, Guzelkucuk U, Yazar F, Tan AK. Accuracy of ultrasound-guided genicular nerve block: A cadaveric study. Pain Physician 2015; 18:E899-E904.

83. Gofeld M. Ultrasonography in pain medicine: A critical review. Pain Pract 2008; 8:226-240.

84. Halyard Health. Nerve ablation by cooled radiofrequency compared to corticosteroid injection for management of knee pain. https://ClinicalTrials.gov/ show/NCT02343003.

85. Liu SS, Buvanendran A, Rathmell JP,
Sawhney M, Bae JJ, Moric M, Perros S, Pope AJ, Poultsides L, Della Valle CJ, Shin NS, McCartney C), Ma Y, Shah M, Wood MJ, Manion SC, Sculco TP. A cross-sectional survey on prevalence and risk factors for persistent postsurgical pain 1 year after total hip and knee replacement. Reg Anesth Pain Med 2012; 37:415-422.

86. Ghogomu EA, Maxwell LJ, Buchbinder R, Rader T, Pardo Pardo J, Johnston RV, Christensen RD, Rutjes AW, Winzenberg TM, Singh JA, Zanoli G, Wells GA, Tugwell P; Editorial Board of the Cochrane Musculoskeletal Group. Updated method guidelines for Cochrane musculoskeletal group systematic reviews and metaanalyses. J Rheumatol 2014; 41:194-205.

87. Staal JB, de Bie RA, de Vet HC, Hildebrandt J, Nelemans P. Injection therapy for subacute and chronic low back pain: An updated Cochrane review. Spine 2009; 34:49-59.

88. National Heart, Lung, and Blood Institute. Quality assessment of controlled intervention studies. www.nhlbi.nih. gov/health-pro/guidelines/in-develop/ cardiovascular-risk-reduction/tools/rct. March 2014. Accessed October 3, 2016.

89. National Heart, Lung, and Blood Institute. Quality assessment tool for beforeafter (pre-post) studies with no control group. www.nhlbi.nih.gov/health-pro/ guidelines/in-develop/cardiovascularrisk-reduction/tools/before-after. March 2014. Accessed October 3, 2016.

90. US Preventive Services Task Force. Grade definitions. www.uspreventiveservicestaskforce.org/Page/Name/ grade-definitions. July 2012. Accessed October 3, 2016. 
